\title{
Imaging Structure and Magnetisation in New Ways Using 4D STEM
}

Ian MacLaren ${ }^{1}$, Magnus Nord ${ }^{1}$, Suzanne Conner ${ }^{1}$, Damien McGrouther ${ }^{1}$, Christopher S. Allen ${ }^{2,3}$, Mohsen Danaie ${ }^{3}$, Angus I. Kirkland ${ }^{2,3}$, Rantej Bali ${ }^{4}$, Gregor Hlawacek ${ }^{4}$, Jürgen Lindner ${ }^{4}$ and Jürgen Faßbender ${ }^{4}$

1. School of Physics and Astronomy, University of Glasgow, Glasgow G12 8QQ, UK

2. Department of Materials, University of Oxford, Parks Road, Oxford OX1 3PH, UK

3. Electron Physical Sciences Imaging Centre (ePSIC), Diamond Light Source, Didcot OX11 0DE, UK

4. Helmholtz-Zentrum Dresden-Rossendorf, Institute of Ion Beam Physics and Materials Research, Bautzner Landstr. 400, D-01328 Dresden, Germany.

Integrating fast readout direct electron detectors into the scanning transmission electron microscope (STEM) is revolutionizing imaging. This paper provides a brief review of work in this area at Glasgow.

The Materials and Condensed Matter group at the University of Glasgow has long been experimenting with direct electron detectors, together with the Particle Physics Experimental group. A Medipix2 detector was integrated onto our Philips CM20FEG electron microscope in a bottom mount position in 2010 and used in initial studies [1,2]. More recently, a Medipix3 detector was installed onto our JEOL ARM200F in a fixed mount at the $35 \mathrm{~mm}$ camera port, and together with a Merlin readout system (Quantum Detectors Ltd., Harwell, UK) it was possible to run 4D STEM imaging at $>1000$ frames per second, under the control of Gatan Digital Micrograph. A similar system has been installed in a bottom mount on the JEOL Grand ARM at ePSIC. Most recently, a retractable mount for the Medipix3 detector has been produced at Glasgow, and is now installed on our JEOL ARM200F in the 35mm port.

One area of use of pixelated detection is for the improved differential phase contrast (DPC) mapping of magnetization in materials. Traditional DPC-STEM uses split detectors to determine the deflection of the bright field disc but suffers from diffraction based intensity variation within the disc leading to spurious unwanted contrast on the length-scale of the grains. This can be overcome using a pixelated detector, and processing the discs using edge detection and cross correlation to give a pure disc deflection without the diffraction contrast [3]. This has been used in studying the magnetic structures arising from the patterning of $\mathrm{B} 2 \mathrm{Fe}_{60} \mathrm{Al}_{40}$ - this structure is ordered and paramagnetic after deposition and annealing, but after exposure to a (suitably low dose) $\mathrm{Ne}^{+}$beam it is disordered to a BCC structure, which is strongly ferromagnetic and has a larger lattice parameter. We recorded diffraction patterns (Fig 1a) at a camera length to show both the central bright field disc and the inner rings of crystal diffraction spots. Fig $1 \mathrm{~b}$ shows the plotted magnetization in one of these stripes. Fig 1c shows the lattice parameters determined from the diffraction ring radii in the directions parallel to and perpendicular to the long stripe axis. It is clear that parallel to the length, the parameter is constrained by the surrounding film, whereas there is a larger expansion perpendicular to the interface, across the width of the patterned magnetic stripe. This suggests that strain can be used to bias the magnetization in films.

Another novel use for pixelated detectors is to use high angle scattering into higher order Laue zones to reveal ordering along the beam direction, $\mathbf{B}$ [4]. Datasets containing such high angle information have now been recorded at atomic resolution, such as Fig 2 from a perovskite oxide heterostructure. Fig 2a shows HAADF contrast for this dataset, and both La-containing layers are much brighter than the $\mathrm{SrTiO}_{3}$ layer, as expected. Fig $2 \mathrm{~b}$ shows the integrated intensity in the inner Laue zone ring, which only 
appears for the $\mathrm{LaFeO}_{3}$ layer. This arises from the doubling of the unit cell along $\mathbf{B}$, and the intensity roughly corresponds to the magnitude of atomic shuffles parallel to $\mathbf{B}$, showing that these only reach a maximum at the layer midpoint. Careful inspection also shows that the signal is strongest on the La columns, demonstrating that they are the primary source of the period doubling. This allows detailed investigation of the local "crystal structure", which differs from the bulk $\mathrm{LaFeO}_{3}$ structure. The plots in Fig 2c show these trends for lines drawn from left to right, together with the outer Laue zone radius, which reveals subtle changes in lattice parameter along the beam direction across this heterostructure.

This demonstrates two exciting areas for application of such fast pixelated detectors for 4D STEM imaging, and shows that it enables new kinds of investigations, not possible with traditional detectors.

\section{References:}

[1] A MacRaighne et al., Journal of Instrumentation 6, (2011) C01047.

[2] R Beacham et al., Journal of Instrumentation 6 (2011) C12052.

[3] M Krajnak et al., Ultramicroscopy 165 (2016) 42

[4] M Nord et al., Microscopy and Microanalysis, 22 [S3] (2016) p. 476.

[5] This work was funded by the EPSRC (Fast Pixel Detectors: a paradigm shift in STEM imaging, EP/M009963/1). We thank Diamond Light Source for access and support in use of the electron Physical Science Imaging Centre (EM16952) that contributed to the results presented here.
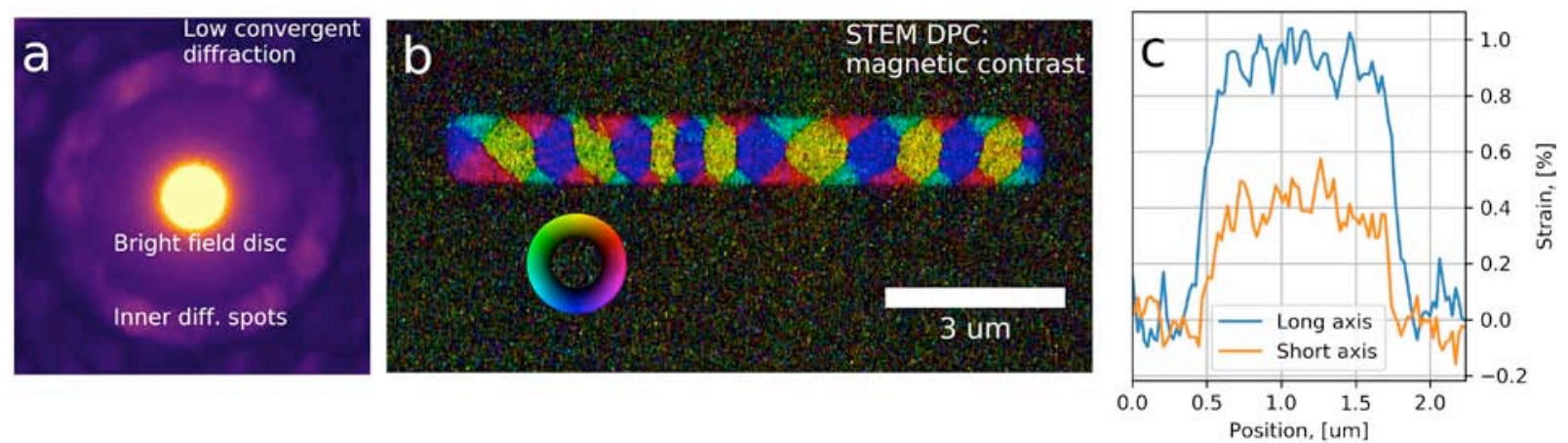

Figure 1. Ion-beam patterning of stripes on $\mathrm{FeAl}$ thin films and the results on magnetization and strain: a) diffraction pattern; b) plot of magnetization determined from disc deflection; c) line plot of the lattice parameters perpendicular and parallel to the long axis of the stripe.
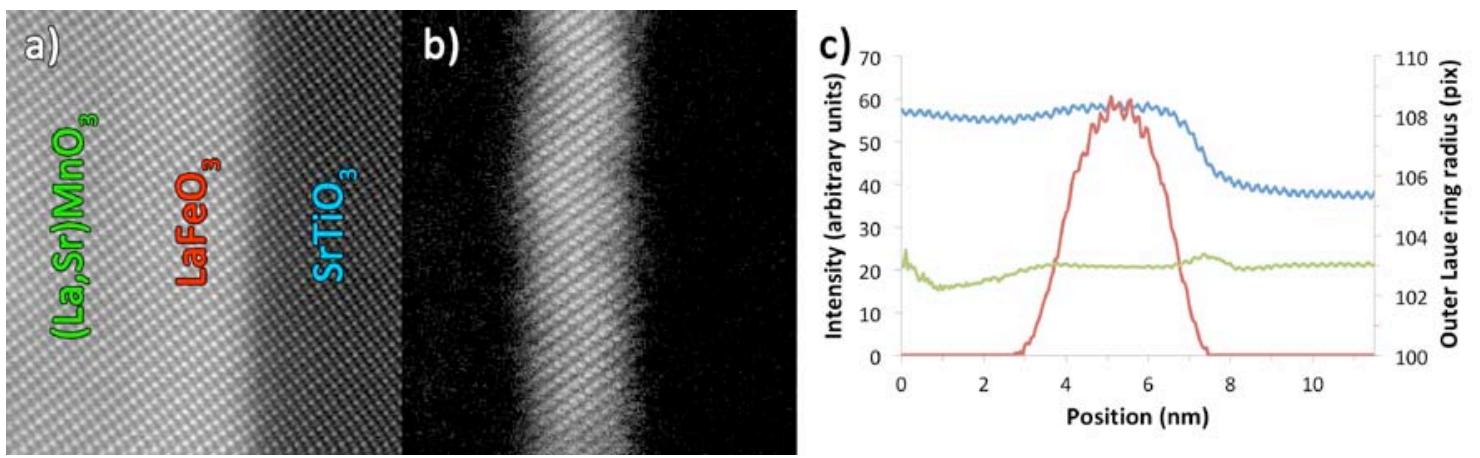

Figure 2. Atomic-resolution Laue zone imaging of a heterostructure of LSMO and LFO on STO: a) HAADF image constructed from the 4D STEM data; b) intensity in the inner Laue zone ring (corresponding to unit cell doubling along the beam direction); c) line plots of ADF intensity (blue), inner Laue ring intensity (red), and outer Laue ring position (green). 